\title{
HyCell: Enabling GREEN Base Station Operations in Software-Defined Radio Access Networks
}

\author{
Tao Zhao, Liumeng Wang, Xi Zheng, Sheng Zhou, and Zhisheng Niu \\ Tsinghua National Laboratory for Information Science and Technology (TNList) \\ Department of Electronic Engineering, Tsinghua University, Beijing 100084, China \\ \{t-zhao12,wlm14, zhengx14\}@ mails.tsinghua.edu.cn, \{sheng.zhou, niuzhs\}@tsinghua.edu.cn
}

\begin{abstract}
The radio access networks (RANs) need to support massive and diverse data traffic with limited spectrum and energy. To cope with this challenge, software-defined radio access network (SDRAN) architectures have been proposed to renovate the RANs. However, current researches lack the design and evaluation of network protocols. In this paper, we address this problem by presenting the protocol design and evaluation of hyper-cellular networks (HyCell), an SDRAN framework making base station (BS) operations globally resource-optimized and energy-efficient (GREEN). Specifically, we first propose a separation scheme to realize the decoupled air interface in $\mathrm{HyCell}$. Then we design a BS dispatching protocol which determines and assigns the optimal BS for serving mobile users, and a BS sleeping protocol to improve the network energy efficiency. Finally, we evaluate the proposed design in our HyCell testbed. Our evaluation validates the feasibility of the proposed separation scheme, demonstrates the effectiveness of BS dispatching, and shows great potential in energy saving through BS sleeping control.
\end{abstract}

Index Terms-Software-defined networking, radio access network, base station sleeping.

\section{INTRODUCTION}

The radio access networks (RANs) are facing great challenges in the mobile Internet era. On one hand, next-generation RANs are expected to support 1000 times increased data traffic [1] with limited spectrum and energy. Therefore, both spectrum efficiency and energy efficiency should be vastly improved. On the other hand, emerging applications and services put forward increasingly diverse requirements for connections in terms of capacity, latency, and reliability. Hence, next-generation RANs need to be sufficiently flexible to accommodate evolving applications.

To tackle these challenges, software-defined networking (SDN) have been proposed to renovate RANs. SoftRAN [2] introduced a logically centralized big base station (BS) to globally optimize the RAN resources so that the network efficiency can be improved. The control-data separation concept of SDN was extended to decouple the control and data coverages at the air interface in RANs [3]-[5]. With the decoupled air interface, BS sleeping control can be effectively employed to reduce energy waste by adapting to real traffic dynamics and improve the network energy efficiency without generating coverage holes. Moreover, CONCERT [6] proposed to deploy RANs as software-defined services, which can dramatically improve the flexibility of BS operations. However, these studies focus on the architecture design only, and leave the protocol design as an open research issue.
Besides design, it is of much research interest to evaluate the SDN concepts in practical RAN implementations. Our previous work presented a prototype system which demonstrated the feasibility of decoupled air interface on top of the GSM standard [7]. But only a single type of service (namely voice calls) was investigated, and no attempt to realize dynamic BS operations was made. PRAN [8] showed a preliminary implementation of dynamic resource allocation of BSs with centralized RAN schedulers. However, other BS operations such as BS sleeping were not studied. To our knowledge, there are few efforts to evaluate the protocols of BS operations in software-defined RANs (SDRANs) with practical implementations.

In this paper, we present the protocol design and evaluation of our SDRAN framework named hyper-cellular networks (HyCell). HyCell enables globally resource-optimized and energy-efficient (GREEN [9]) BS operations by exploiting the decoupled air interface, centralized BS control, and softwaredefined BS functions. To design the decoupled air interface, we take an evolutionary approach and propose our separation scheme for current 3GPP standards, which is beneficial for network migration. Based on that, we design our BS dispatching protocol, which determines and assigns the globally optimal BS to serve the user requests, as well as our BS sleeping protocol. Moreover, we prototype a HyCell testbed on a software-defined radio (SDR) platform, and use it to evaluate our design. The main contributions of our work are summarized as follows:

1) We propose a separation scheme to realize the decoupled air interface for existing 3GPP standards from the aspects of both network functionalities and logical channels, and demonstrate its feasibility in the testbed.

2) We design a BS dispatching protocol for global optimization of network resources, and implement a BS dispatching scheme to effectively achieve load balancing among multiple BSs.

3) We design a BS sleeping protocol to improve the network energy efficiency, and present an implementation with a threshold-based algorithm, which shows about $60 \%$ energy saving gain in our testbed.

The rest of this paper is organized as follows. We first give an overview of the HyCell architecture as well as the challenges and solutions to realize it in Section III. Then we 


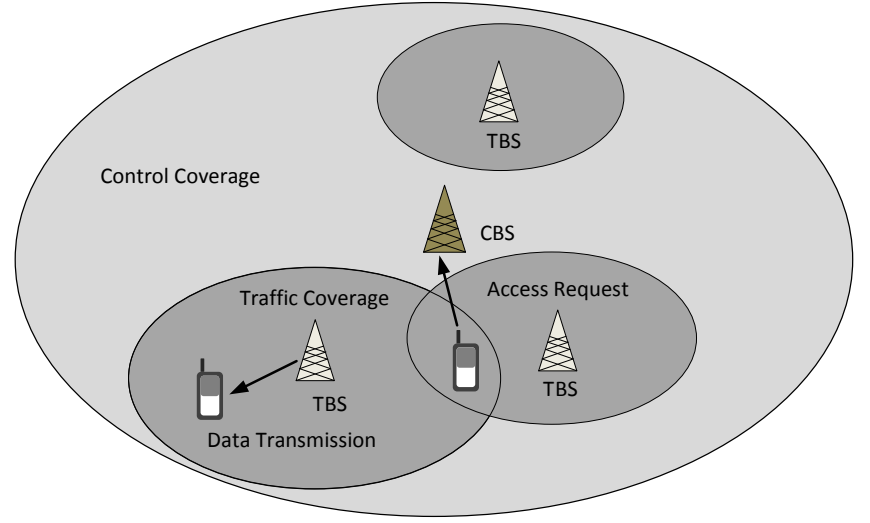

Fig. 1. HyCell architecture.

present the design of the separation scheme, BS dispatching, and BS sleeping in Section III Evaluation of the testbed implementation is given in Section IV Section $\mathrm{V}$ concludes the paper.

\section{OVERVIEW}

\section{A. Architecture}

As illustrated in Fig. 1. HyCell decouples the air interface of the RAN by separating the control coverage from the traffic coverage with two types of BSs. Control BSs (CBSs) provide the control coverage, while traffic BSs (TBSs) provide the traffic coverage. Typically CBSs have large coverage areas, within which multiple traffic BSs (TBSs) are deployed. CBSs provide the network control to underlying TBSs and mobile users. In particular, they grant the network access to mobile users. To guarantee a basic level of data service and to cope with high mobility users, CBSs can also be used to provide low-rate data services such as voice call service to the UE. Unlike CBSs, TBSs are only responsible for high-rate data services. Specifically, there can be different subtypes of TBSs to support different classes of high-rate data services. Through the separation, CBSs and TBSs can be more simplified than conventional BSs.

Under the separation architecture, CBSs provide centralized control, and enable dynamic operations of TBSs. CBSs and TBSs serve mobile users collaboratively. When the user equipment (UE) is powered on, it searches for nearby CBSs and registers to the network through a CBS. CBSs gather network state information such as UE locations and traffic load from the mobile users and TBSs underlying its coverage, and thus hold a global view of the network. When high-rate data services are required, the UE sends requests to the CBS and the CBS dispatches one or more TBSs for the high-rate data transmission afterwards. When the network traffic load is light, TBSs can be turned off under the command of the CBS to reduce the energy consumption of the network while the CBS preserves the network coverage.

Moreover, HyCell decouples the software which realizes the BS functions from the hardware which converts signals between baseband and radio frequency. In this way, BS functions are software defined by high-level programming languages, and it is much easier to update the BSs to handle new mobile Internet applications and services. Further, with the help of virtualization, BSs can become virtual instances in cloud data centers. Network efficiency can be improved with resource pooling, and BS operations can be more flexible with the help of virtual machine migration.

With the decoupled air interface, centralized BS control, and software-defined BS functions, HyCell enables GREEN BS operations in SDRANs. It leads to a promising path towards next-generation RANs.

\section{B. Challenges and Solutions}

We summarize the challenges of protocol design in HyCell and our proposed solutions as follows.

a) Separation of Air Interface: In this paper, we target at current air interface protocols and propose our separation scheme to design a decoupled air interface. However, it is a daunting task to analyze the air interface in existing 3GPP standards. The physical layer signals are difficult to categorize and the interactions among them are complicated, making it difficult to separate the control coverage from the traffic coverage. To tackle this challenge, we propose our separate scheme from the aspects of network functionalities and logical channels, rather than physical layer signals. In our design, we jointly consider the two levels and ensure that the expected network functionalities of CBSs and TBSs can be mapped to their corresponding logical channels. This guides us to an effective separation scheme of the air interface.

b) CBS-TBS Protocol Design: The CBS-TBS protocols do not exist in current 3GPP standards. However, the protocols are crucial for the SDRANs to optimize the network performance. Specifically, we need a protocol to find the best TBSs and assign them to serve the UE. We also need a protocol to dynamically switch off TBSs to reduce energy waste and improve network energy efficiency. The signaling interactions in the protocols must carry sufficient network state information for global optimization. At the same time, it should ensure realtime decision and accommodate potential updates. To meet the requirements, we choose simple but effective metrics of the network state information in message exchange. We also make our protocols extensible by adopting modular design.

c) UE Transparency: When designing HyCell, we would like to guarantee that the updates at the BS side are transparent to the UE side. With UE transparency, the existing mobile terminals can work with HyCell automatically. It brings the benefit of compatibility, which is appealing during the protocol evolution and network upgrade. However, it also limits the degree of freedom we have to realize the SDRAN. To achieve UE transparency, we carefully design the separation scheme of the air interface and make sure that the UE-facing interfaces are preserved. In addition, we exploit the backhaul connection for CBS-TBS communication and cooperation, which optimizes the network without the need of adding functions to mobile terminals. 
TABLE I

LOGICAL CHANNEL SEPARATION OF 3GPP STANDARDS.

\begin{tabular}{c|c|c|c|c}
\hline \multicolumn{3}{|c|}{ Logical Channel } & \multicolumn{2}{c}{ Separation } \\
\hline GSM/GPRS & UMTS & LTE & CBS & TBS \\
\hline BCH & BCCH & BCCH & $\checkmark$ & \\
\hline CCCH & CCCH, PCCH & CCCH, PCCH & $\checkmark$ & \\
\hline PACCH & DCCH & DCCH & & $\checkmark$ \\
\hline TCH & CTCH & MTCH, MCCH & $\checkmark$ & \\
\hline PDTCH & DTCH & DTCH & & $\checkmark$ \\
\hline
\end{tabular}

\section{Key Design Aspects}

In this section we describe our key design aspects. First we analyze the air interface of 3GPP standards and propose a separation scheme for HyCell. Then a BS dispatching protocol design is proposed, and we also present a BS sleeping protocol which achieves great energy saving gain without sacrificing mobile users' quality of service.

\section{A. Separation of Air Interface}

First, we present our separation scheme of the air interface from the view of network functionalities. We categorize network functionalities into five classes: synchronization, broadcast of system information, paging, low-rate data transmission, and high-rate data transmission. To realize the decoupled air interface of HyCell, we need to separate the network functionalities into two types of BSs. In our design, CBSs are in charge of synchronization, broadcast of system information, paging, and low-rate data transmission, while TBSs are only responsible for high-rate data transmission.

Next, we propose our separation scheme from the perspective of logical channels considering their corresponding network functionalities. The logical channels of 3GPP standards can be classified into five groups, while the specific channel names vary in different standards [10]. As shown in Table I] BCCH (broadcast control channel, $\mathrm{BCH}$ in GSM/GPRS) and $\mathrm{CCCH}$ (common control channel, including PCCH for paging in UMTS and LTE) are reserved by CBSs since they are in charge of network functionalities including synchronization, paging, and broadcast of system information. CTCH (common traffic channel, TCH in GSM/GPRS, MTCH and MCCH for multicast in LTE) is also left at the CBS side to take care of low-rate data transmission. At the TBS side are DTCH (dedicated traffic channel) and DCCH (dedicated control channel), which are responsible for high-rate data transmission. In the case of GSM/GPRS, we use PDTCH (packet data traffic channel) and PACCH (packet associated control channel) for packet data transmission and associated control.

Compared with the separation scheme of the GSM standard [7], here we differentiate the low-rate data service from the high-rate data service, which results in a distinct separation scheme of logical channels. This more fine-grained separation allows the network to ensure a basic level of data service to the mobile users, and thus is more practical. Unlike the functionality separation scheme in existing work [11], we also present the separation scheme from the aspect of logical channels. Besides, because of the joint consideration of network functionalities and logical channels, synchronization in the air interface resides only at the CBS side in our design. We will show in Section IV that our separation scheme is feasible as long as fine synchronization between CBSs and TBSs is guaranteed.

\section{B. Base Station Dispatching}

Through the separation of the air interface, the control coverage and the traffic coverage of the RAN are decoupled. The CBS can gather the information of all mobile users and TBSs in its coverage, and thus have a global view of the network. Based on that, the CBS can dispatch one or more TBSs to serve the high-rate data service request of mobile users in a globally optimal fashion. To this end, a protocol of BS dispatching is needed.

One possible design of BS dispatching is based on channel state information (CSI) between the UE and the TBSs. If we collect the CSI and make it available to the CBS, it can calculate the achieved data rate with each TBS, and choose the TBS with the highest value. However, it is difficult to acquire the CSI since the TBSs might have no prior connection to the UE. One possible solution is that we embed pilot symbols in the channel requests. Then we demand that TBSs monitor the channel requests and report the measured CSI to the CBS. But besides the potential breakage of UE transparency, large quantity of CSI also poses a heavy burden on the backhaul link, for instance, the X2 interface in LTE, especially in multiple antenna scenarios.

A more practical design is based on traffic load. The load information can be readily acquired at each TBS, and aggregated at the CBS. It is also an important metric to characterize the network state. In a heavily-loaded network, balancing the load of multiple TBSs is beneficial because it can potentially deliver better service in addition to improving radio resource utilization and reducing the risk of system failure ${ }^{1}$

Fig. 2 illustrates the signaling interaction of the loadbalancing BS dispatching protocol. When the TBSs are actively serving the mobile users, they send to the CBS a TBS load information message in packets through the backhaul link. The packet header contains a label to indicate the message type, the TBS ID, and the timestamp of packet transmission. The packet payload is related to the specific air interface. For example, with the decoupled GSM/GPRS air interface, the payload includes the time slot usage, the absolute radiofrequency channel number (ARFCN) usage, and the data transmission rate. After receiving the message, the CBS reserves the statistical data for a preset period. When a new channel request of high-rate data service arrives, the CBS computes the load of each TBS, and chooses the least loaded TBS to provide the service. Then the CBS sends a TBS request message to the chosen TBS, and waits for a response message with new

\footnotetext{
${ }^{1}$ In the lightly-loaded scenario, BS sleeping is preferable, and the protocol will be described in Section III-C
} 


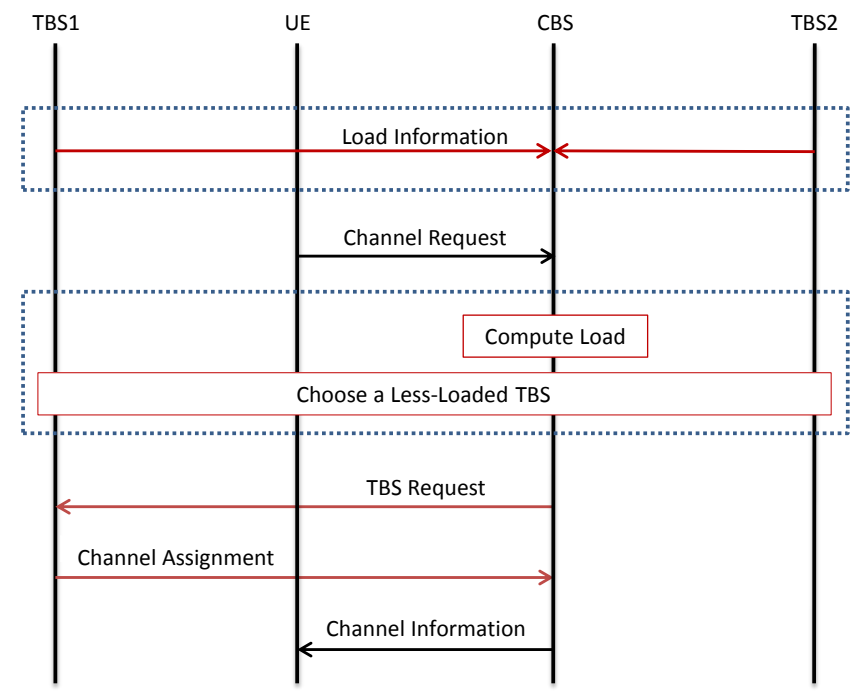

Fig. 2. Signaling interaction of BS dispatching.

channel assignment. After that the CBS informs the user of the assigned channel, and the link between the TBS and the mobile user is established.

\section{Base Station Sleeping}

With the decoupled air interface, CBSs can provide an "always-on" control coverage, and we can turn TBSs into sleep mode flexibly and dynamically without generating unwanted coverage holes. Because the CBS hold the global network state information in its coverage, it can decide which TBSs go to sleep to improve the network energy efficiency.

Next we introduce the protocol of BS sleeping in HyCell. Assume the network load is light, and not all TBSs are needed to meet the mobile users' service requirement. The CBS periodically executes a BS sleeping control routine which determines the modes of the underlying TBSs to maximize the network energy efficiency. In each cycle, it sends a BS sleeping message to each active TBS to be turned off, and a BS waking up message to each sleeping TBSs to be turned on. The BS sleeping and waking up messages are transmitted through the backhaul link between CBSs and TBSs. They require acknowledgment to ensure robust control of BS sleeping.

Thanks to the software-defined nature of BS functions, We can adopt modular design, and make the API of the BS sleeping control routine independent of the specific algorithm inside. In this way, the algorithm involved can be updated without changing the overall protocol, and thus the protocol is extensible. Several BS sleeping algorithms have been developed in the literature [12]-[15]. Existing centralized algorithms for conventional RANs can be put into use in the SDRANs by assuming the controller to be at the CBS. While centralized solutions is favored because of the optimality guarantee and the ease to develop and implement, it might not suit all needs. For instance, when TBSs are much densely deployed compared with CBSs, the centralized decision could result

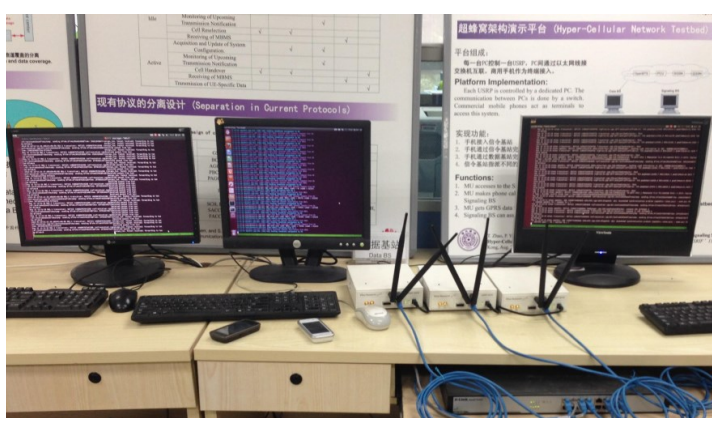

Fig. 3. HyCell testbed.

TABLE II

BSS AND PCS IN THE HYCELL TESTBED.

\begin{tabular}{lll}
\hline BS & PC Model & Processor \\
\hline CBS & Dell Optiplex 990 & Intel Core i7-2600 \\
TBS1 & Dell Optiplex 755 & Intel Core2 Duo E6550 \\
TBS2 & Dell Optiplex 9010 & Intel Core i7-3770 \\
\hline
\end{tabular}

in prohibitively high complexity. In such case, decentralized control or distributed algorithms can be utilized to reach a better tradeoff.

\section{Evaluation}

\section{A. Testbed Setup}

As shown in Fig. 3, we utilize an SDR platform to prototype the HyCell testbed, which can demonstrate GREEN BS operations in SDRANs. In this testbed, we employ three commodity PCs to run the software applications of one CBS and two TBSs. The relevant parameters are listed in Table II We use two commercial mobile phones (Nokia 5230) to test the network. In our tests, the two TBSs have overlapping traffic coverage, where a mobile user can be served by either TBS as in Fig. 1. Note that it is straightforward to add more TBSs to the testbed by setting up more PCs with the TBS software.

We use USRP N210s with WBX daughter boards and VERT900 antennas from Ettus Research 2 as the hardware to transform data between radio frequency and baseband. The USRP N210s and PCs are all connected to a Gigabit Ethernet switch. We install the GPS-disciplined oscillators (GPSDO) module to each N210. Each GPSDO outputs a $10 \mathrm{MHz}$ reference and a pulse-per-second (PPS) signal, which provide frequency and time reference for all BSs [16]. In addition, the UE is synchronized with the CBS through the air interface. Therefore, we can achieve fine synchronization between the UE, the associated CBS, and the serving TBS, which serves as a base to provide a seamless service flow under the separation architecture.

The BS applications are all running on Ubuntu GNU/Linux 12.04.1 LTS. We developed our CBS and TBS applications based on a GPRS fork of OpenBTS P2.8 $3^{3}$ and Osmocom ${ }^{4}$

\footnotetext{
2 http://www.ettus.com

3 https://github.com/chemeris/openbts-p2.8

${ }^{4}$ http://osmocom.org/
} 

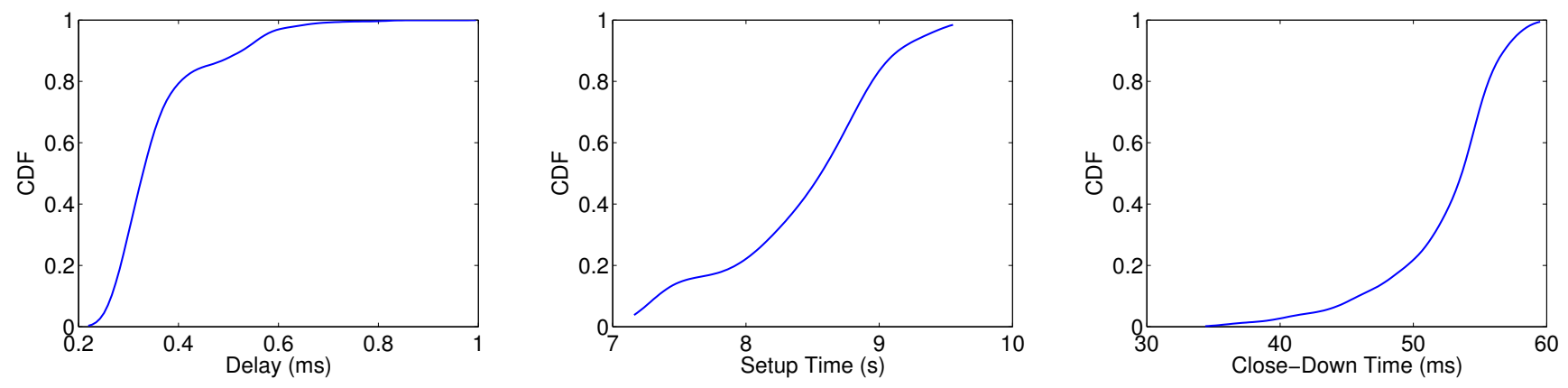

Fig. 4. CDF of (a) delay overhead, (b) setup time, and (c) close-down time.

components including osmo-pcu, OsmoSGSN, and OpenGGSN. Besides, Asterisk 5 is used to connect phone calls.

\section{B. Feasibility of Separation}

We implement our proposed separation scheme of the GSM/GPRS air interface in the testbed to prove the feasibility of separation. In our tests, the CBS provides network coverage, voice call service, and short message service (SMS) for UE, while UE can acquire GPRS data service from the TBSs. Specifically, after the UE sends a channel request to the CBS, the CBS analyzes the requested channel type. If the channel type is PDTCH, the CBS dispatches a TBS to serve the UE. The messages between the CBS and the TBS are transmitted through the wired connection with UDP packets. Afterwards, packet-switched data transmission is established between the TBS and the UE.

In HyCell, the signaling interaction between the CBS and the TBS incurs an additional delay when processing the channel request of high-rate data service. We term it as the "delay overhead" of separation. We measured the delay overhead in our tests, and plot its distribution in Fig. 4a The mean value of the signaling overhead in our testbed is about $0.36 \mathrm{~ms}$ and the standard variance is about $0.1 \mathrm{~ms}$. The overhead is quite small and can be negligible compared to the time that the UE waits before retransmitting the channel request. Besides, in terms of user experience, there is no noticeable service quality degradation in our tests. Therefore, we validates the feasibility of the separation scheme in our testbed.

\section{Effectiveness of Load Balancing}

We put the load-balancing dispatching scheme in Section III-B into practice and evaluated its performance in the tests. In the implementation we measure the quantity of data transmitted on PDTCH and use it to calculate the traffic load of each TBS. We do not include ARFCN usage in the packet in the testbed implementation since each TBS only has one preconfigured radio carrier.

In our test scenario, initially a mobile user is served by a single TBS. Then we activate another TBS and mobile phone. At this time, the second TBS has no traffic load. Afterwards,

\footnotetext{
5 http://www.asterisk.org/
}

TABLE III

POWER CONSUMPTION COMPARISON. (UNIT: W)

\begin{tabular}{c|c|c|c}
\hline Component & No Sleep & Half Sleep & Full Sleep \\
\hline PC & 346 & 310 & 129 \\
\hline USRP & 41.4 & 13.8 & 13.8 \\
\hline Switch & 21 & 21 & 21 \\
\hline Total & 408.4 & 344.8 & 163.8 \\
\hline
\end{tabular}

the CBS will assign the second TBS to serve the channel requests of the new mobile user, which drives the load of the two TBSs to approach equal. The results demonstrate that the scheme achieves load balancing of multiple TBSs.

\section{Base Station Sleeping}

We implement the protocol of TBS sleeping with a simple threshold-based algorithm in our testbed. In the implementation, sleeping and waking up of the TBSs are decided by the CBS considering the total traffic load of the TBSs. A sleeping and waking up module is created at each TBS, which receives the sleeping or waking up command from the CBS, and turns off or turns on the TBS by starting or stopping the TBS application accordingly. As the on and off states of the USRPs cannot be controlled by the baseband software, we need to power on or power off the USRPs manually.

We measured the setup and close-down time of BS sleeping, and show their distributions in Fig. $4 \mathrm{~b}$ and Fig. $4 \mathrm{c}$ respectively. The average close-down time when turning off a TBS is $52 \mathrm{~ms}$, while the average setup time when waking up a TBS, during which the TBS is initialized and synchronized, is $8.4 \mathrm{~s}$. Note that the setup time is rather long, making the current BS sleeping and waking up implementation unsuitable for services that require low latency. Further work on reducing the setup time is thus necessary.

To show the energy saving performance of BS sleeping, we currently take a component analysis approach due to the lack of power measurement equipment. Based on the specifications, we calculate the power consumption of PCs, USRPs and the switch respectively and take the sum as the total power consumption of the testbed. Table III compares the power consumption of different sleep modes in the scenario when there 
are no active user requests. Without BS sleeping (No Sleep in the table), all BSs are idle, and the total power consumption is $408.4 \mathrm{~W}$. When we only turn off the USRPs and software BSs on PCs (Half Sleep), the total power consumption is $344.8 \mathrm{~W}$, that is, $16 \%$ power consumption can be saved. If we further power off the PCs (Full Sleep), the total power can be reduced to $163.8 \mathrm{~W}$, which translates to $60 \%$ energy savings. It is thus observed that BS sleeping can bring significant energy saving gain, of which the major part comes from the baseband power consumption.

\section{CONCLUSiON}

In this paper we present the design and evaluation of HyCell. The proposed framework HyCell exploits the decoupled air interface, centralized BS control, and software-defined BS functions to enable GREEN BS operations in SDRANs. We propose a separation scheme to realize the decoupled air interface, and further design the protocols of BS dispatching and BS sleeping. Our testbed implementation validates the feasibility of the separation scheme for the GSM/GPRS standard. Further the test results show that BS dispatching can effectively achieve load-balancing, and BS sleeping can provide about $60 \%$ energy saving gain.

\section{ACKNOWLEDGMENT}

We would like to thank Yuyang Wang and Guangchao Wang for their help in the evaluation. We would also like to thank Dr. Xu Zhang, Xueying Guo, Jingchu Liu, and anonymous reviewers for their insightful comments. This work is sponsored in part by the National Basic Research Program of China (No. 2012CB316001), and the National Natural Science Foundation of China (NSFC) under grant No. 61201191 and 61401250, the Creative Research Groups of NSFC (No. 61321061), the SinoFinnish Joint Research Program of NSFC (No. 61461136004), and Intel Corporation.

\section{REFERENCES}

[1] 4G Americas, "Meeting the 1000x challenge: The need for spectrum, technology and policy innovations," White Paper, May 2014, (Condensed Version). [Online]. Available: http://www.4gamericas.org/ index.php/download_file/view/208/159/

[2] A. Gudipati, D. Perry, L. E. Li, and S. Katti, "SoftRAN: Software defined radio access network," in Proc. 2nd ACM SIGCOMM Workshop Hot Topics in Software Defined Networking (HotSDN '13), Hong Kong, China, Aug. 2013, pp. 25-30.

[3] Z. Niu, S. Zhou, S. Zhou, X. Zhong, and J. Wang, "Energy efficiency and resource optimized hyper-cellular mobile communication system architecture and its technical challenges," SCIENTIA SINICA Informationis, vol. 42, no. 10, pp. 1191-1203, 2012, (In Chinese).

[4] A. Capone, A. Fonseca dos Santos, I. Filippini, and B. Gloss, "Looking beyond green cellular networks," in 9th Annu. Conf. Wireless OnDemand Network Systems and Services (WONS), Jan. 2012, pp. 127130.

[5] H. Ishii, Y. Kishiyama, and H. Takahashi, "A novel architecture for LTE-B: C-plane/U-plane split and Phantom Cell concept," in IEEE GLOBECOM Int. Workshop Emerging Technologies for LTE-Advanced and Beyond-4G, Dec. 2012, pp. 624-630.

[6] J. Liu, T. Zhao, S. Zhou, Y. Cheng, and Z. Niu, "CONCERT: A cloudbased architecture for next-generation cellular systems," IEEE Wireless Commun. Mag., vol. 21, no. 6, pp. 14-22, Dec. 2014.
[7] T. Zhao, P. Yang, H. Pan, R. Deng, S. Zhou, and Z. Niu, "Software defined radio implementation of signaling splitting in hyper-cellular network," in Proc. 2nd Workshop Software Radio Implementation Forum, in conjunction with ACM SIGCOMM 2013, Hong Kong, China, Aug. 2013, pp. 81-84.

[8] W. Wu, L. E. Li, A. Panda, and S. Shenker, "PRAN: Programmable radio access networks,' in Hotnets '14, Los Angeles, CA, USA, Oct. 2014, pp. 6:1-6:7.

[9] Z. Niu, "TANGO: Traffic-aware network planning and green operation," IEEE Wireless Commun. Mag., vol. 18, no. 5, pp. 25-29, 2011.

[10] M. Sauter, From GSM to LTE: An Introduction to Mobile Networks and Mobile Broadband. John Wiley \& Sons, 2011.

[11] X. Xu, G. He, S. Zhang, Y. Chen, and S. Xu, "On functionality separation for green mobile networks: Concept study over LTE," IEEE Commun. Mag., vol. 51, no. 5, pp. 82-90, 2013.

[12] S. Zhou, J. Gong, Z. Yang, Z. Niu, and P. Yang, "Green mobile access network with dynamic base station energy saving," in Proc. ACM MobiCom'09, Beijing, China, Sep. 2009, (Poster Paper).

[13] X. Guo, S. Zhou, Z. Niu, and P. R. Kumar, "Optimal wake-up mechanism for single base station with sleep mode," in Proc. 2013 25th Int. Teletraffic Congr. (ITC), Sep. 2013.

[14] Y. S. Soh, T. Q. Quek, M. Kountouris, and H. Shin, "Energy efficient heterogeneous cellular networks," IEEE J. Sel. Areas Commun., vol. 31 , no. 5, pp. 840-850, May 2013.

[15] S. Zhang, J. Wu, J. Gong, S. Zhou, and Z. Niu, "Energy-optimal probabilistic base station sleeping under a separation network architecture," in 2014 IEEE Global Communications Conf. (GLOBECOM), Dec. 2014.

[16] UHD, Synchronization Application Notes, 2014. [Online]. Available: http://files.ettus.com/manual/page_sync.html 